\title{
Ruptured Distal Anterior Cerebral Artery Aneurysms Presenting as Acute Subdural Hematoma
}

\section{-Report of Three Cases-}

\author{
Kunihiko Watanabe, Susumu WaKaI, \\ Soushi OKUHATA and Masakatsu NAGAI \\ Department of Neurosurgery, Dokkyo University School of Medicine, Tochigi
}

\begin{abstract}
The authors report three cases of distal anterior cerebral artery aneurysm presenting as acute subdural hematoma (SDH). Two patients were comatose on admission and died of massive SDH. One patient underwent aneurysmal neck clipping in the chronic stage and returned to normal daily life. A convexity SDH continuous with a wedge-shaped interhemispheric SDH was the characteristic computed tomographic appearance in all cases. There was no accompanying subarachnoid or intracerebral hemorrhage in one case (pure SDH). These cases are $9.4 \%$ of 32 ruptured distal ACA aneurysms treated in our institute in the last 14 years, a higher incidence than reported previously.
\end{abstract}

Key words: cerebral aneurysm, distal anterior cerebral artery, subdural hematoma

\section{Introduction}

Ruptured cerebral berry aneurysms presenting as subdural hematoma (SDH) are rare, representing only $0.5-2 \%$ of all ruptured aneurysms in several large series, since computed tomographic (CT) scanning became available. ${ }^{1,2,11,16,18)}$ Fox $^{8)}$ analyzed 148 aneurysmal SDH cases reported between 1895 and 1978. Most SDH originated from the internal carotid artery $(43 \%)$, middle cerebral artery (MCA) $(22 \%)$ and anterior communicating artery (15\%). Only 11 cases originated from aneurysms on the distal anterior cerebral artery (ACA). ${ }^{3-7,9,10,12,17)}$ Five cases have since been reported..$^{1,11,13,20)}$ Here we report three cases of distal ACA aneurysm presenting as SDH and discuss their clinicoradiographic features.

\section{Case Reports}

Case 1: A 51-year-old male lost consciousness suddenly on June 18,1988 . He did not sustain head injury but had a history of severe headache persisting for several days 2 months before. He was admitted 1 hour after the ictus.

Received August 3, 1990; Accepted January 10, 1991
On admission, he was comatose with decerebrate posture. Both pupils were dilated and the light reflex was absent. Bilateral corneal reflex was elicited but the oculocephalic reflex was absent. He breathed spontaneously though ataxic. Precontrast CT scans revealed a massive SDH on the left convexity hemisphere continuous with a wedge-shaped interhemispheric SDH (Fig. 1A).

The SDH was evacuated through an emergency large left frontotemporoparietal craniectomy 1 hour after admission. No cerebral contusion or subarachnoid hemorrhage (SAH) was observed over the hemisphere. Massive arterial bleeding from the interhemispheric fissure was encountered during hematoma evacuation near the midline. The bleeding source within the interhemispheric fissure near the falcine edge was electrocoagulated with a bipolar forceps. A ruptured distal ACA aneurysm was suspected. He never regained consciousness and died the following day.

At autopsy, an aneurysm was found on the pericallosal-callosomarginal artery bifurcation of the left ACA (Fig. 1B). The fundus was electrocoagulated. Marked diffuse brain swelling, and bilateral transtentorial and tonsillar herniations were revealed. No definite SAH was observed.

Case 2: A 72-year-old female suffered a sudden onset 

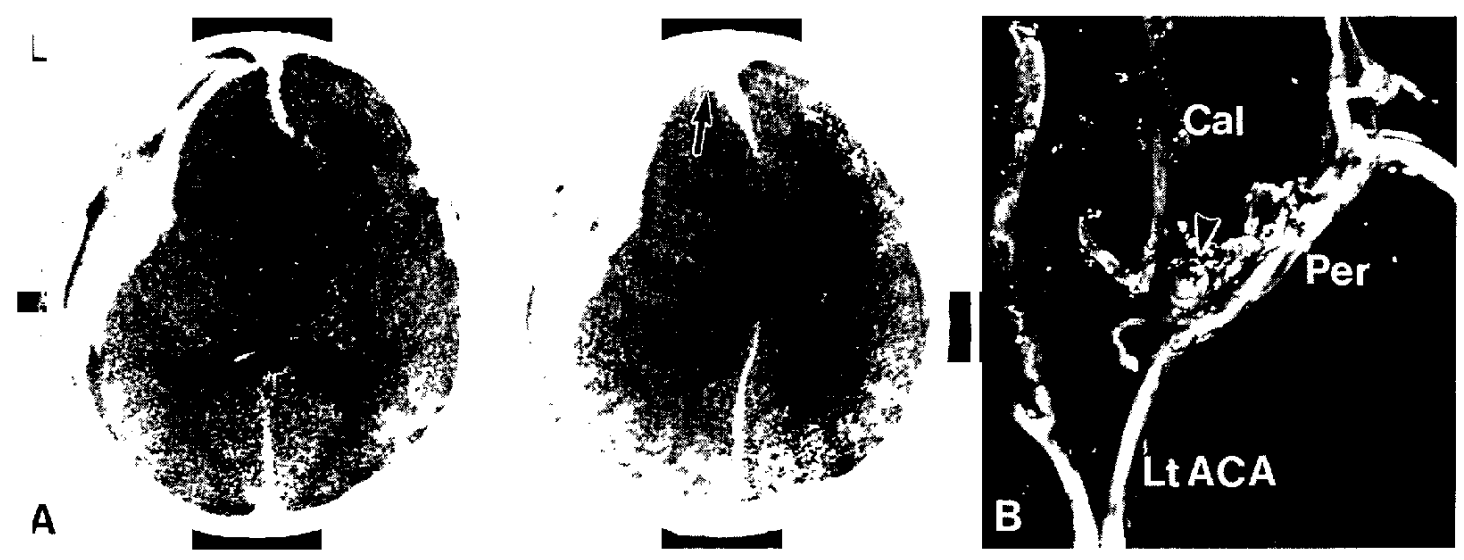

Fig. 1 Case 1. A: Precontrast CT scans showing a large mixed-density crescent SDH continuous with a wedge-shaped interhemispheric SDH (arrow). The midline structures markedly shifted to the right but no subarachnoid hemorrhage is shown. B: Dissected autopsy specimen seen from the base. Arrowhead indicates a ruptured aneurysm on the bifurcation of the callosomarginal (Cal) and pericallosal (Per) arteries of the left anterior cerebral artery (Lt ACA).
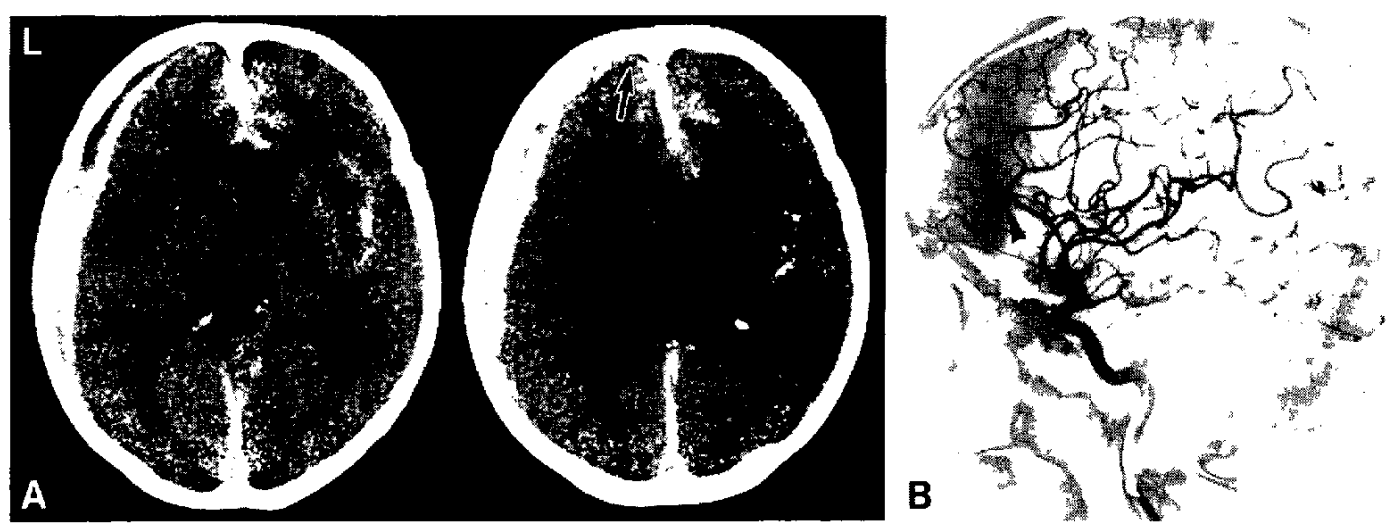

Fig. 2 Case 2. A: Precontrast CT scans revealing a mixed-density crescent SDH continuous with a wedge-shaped interhemispheric SDH (arrow) and diffuse SAH. B: Angiogram showing an aneurysm on the right frontopolar-pericallosal artery bifurcation (arrowhead).

of headache on July 30, 1989. Two hours after the ictus, she was admitted in an obtunded state with right hemiparesis.

Precontrast CT scans showed a crescent SDH on the left convexity continuous with a wedge-shaped interhemispheric SDH and also diffuse SAH (Fig. 2A). These findings suggested a ruptured ACA aneurysm. Angiography demonstrated an aneurysm on the frontopolar-pericallosal artery bifurcation of the right ACA (Fig. 2B).

We decided to operate in the chronic stage because of her age and the absence of increased intracranial pressure signs. The SDH did not increase in size. On day 15 , the aneurysm was clipped. The aneurysm protruded from underneath the falcine edge into the contralateral interhemispheric fissure.

She could walk unaided 2 weeks after surgery and returned to normal daily life.

Case 3: A 74-year-old female lost consciousness and was admitted on January 22, 1990. On admission, she was in a deep coma with decerebrate posture. Respiration was ataxic. The left pupil was dilated and light reflex was absent bilaterally. Precontrast CT scans demonstrated crescent convexity SDH combined with interhemispheric SDH and SAH (Fig. 3A). A distal ACA aneurysm was suspected. Angiography failed as the contrast medium did not penetrate intracranially. She died 3 days after onset. 

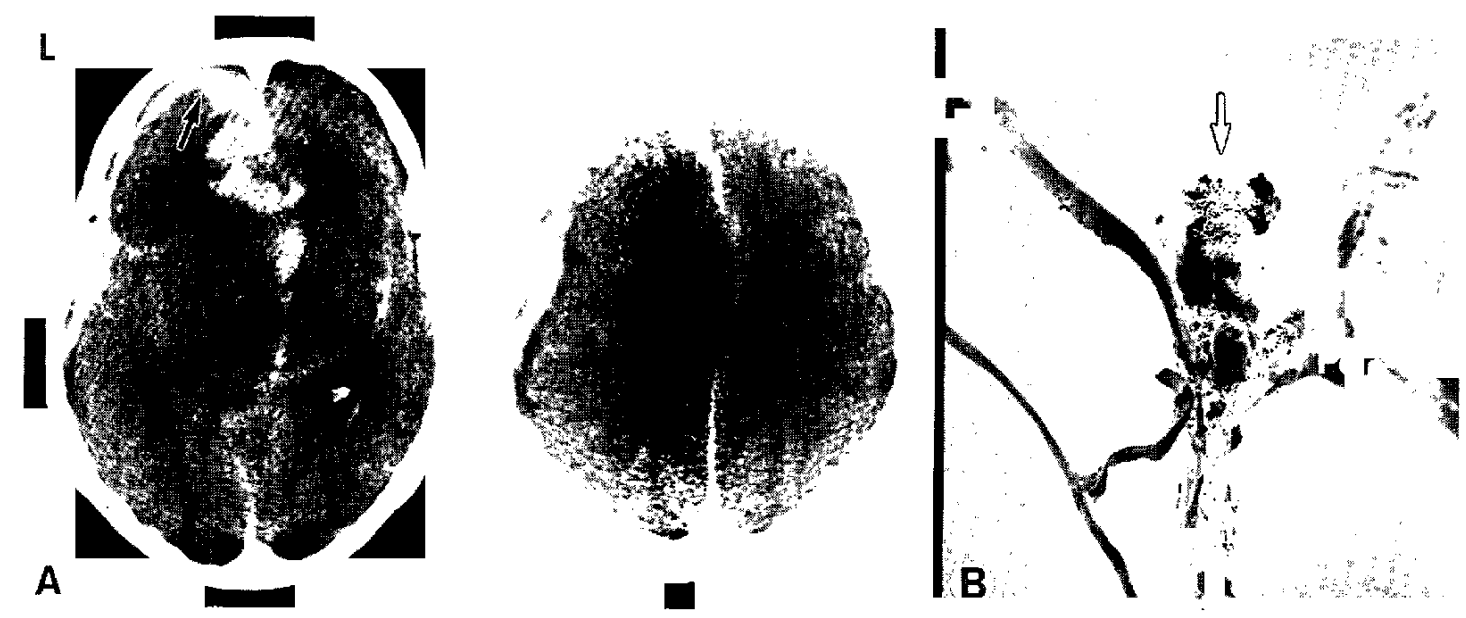

Fig. 3 Case 3. A: Precontrast CT scans demonstrating a mixed-density crescent SDH continuous with a wedge-shaped interhemispheric SDH (arrow). Diffuse subarachnoid hemorrhage is also seen. B: Autopsy specimen viewed from the base showing an aneurysm (arrow) on the frontopolar (Fp)pericallosal (Per) artery bifurcation of the left anterior cerebral artery (Lt ACA).

At autopsy, a ruptured distal ACA aneurysm was observed on the left frontopolar-pericallosal artery bifurcation (Fig. 3B). SDH accompanied by SAH extended from the interhemispheric fissure to the convexity surface.

\section{Discussion}

SDH of non-traumatic origin may be due to cerebral aneurysm rupture. ${ }^{10,16,18)}$ If a convexity SDH is continuous with interhemispheric fissure SDH as seen on the CT scans of our cases, it is highly likely that a ruptured distal ACA aneurysm is the cause. Hemorrhage of arterial origin occurring in the narrow interhemispheric space would be forced to extend over the convexity surface causing this unique CT feature. The possibility should be considered even in pure $\mathrm{SDH}$ as in Case 1, though this is exceptionally rare. ${ }^{14,15)}$ We based our diagnoses for Cases 2 and 3 of a ruptured distal ACA aneurysm causing SDH on the CT appearance previously observed for Case 1.

Weir et al ${ }^{18)}$ proposed the following CT criteria to differentiate SDH caused by ruptured aneurysms or trauma: 1) SDH of aneurysmal origin is unilateral, hyperdense and crescentic in convexity or triangular over the lower Sylvian fissure, while traumatic SDH is more likely to be mixed, iso- or hypodense, may be bilateral or lentiform as well as crescentic, and 2) SDH due to ruptured aneurysms is frequently associated with SAH, intracerebral hemorrhage, or intraventricular hemorrhage while traumatic SDH may be pure SDH or associated with hemorrhagic contusions with further evidence of scalp or bone injury.
The CT appearance of Case 1 fits the criteria for traumatic SDH better than those for aneurysmal origin although there was no scalp or bone injury. The SDH in these three cases appeared as a mixed density on CT scans taken several hours after bleeding, respectively. Thus, the $\mathrm{CT}$ density of a $\mathrm{SDH}$ is probably more correlated with the time after bleeding than with the cause.

We also experienced two SDH patients with a ruptured aneurysm at the MCA. These five SDH cases were about $1.1 \%$ of the 473 aneurysm cases treated at our institute in the last 14 years. This is comparable with other large aneurysm series published recently. ${ }^{1,2,11,16,18,19)}$ Distal ACA aneurysm causes SDH infrequently, but as the distal ACA is an uncommon site for aneurysms ${ }^{191}$ the SDH occurrence might be underestimated. In our series, three of 32 $(9.4 \%)$ distal ACA aneurysms were associated with $\mathrm{SDH}$. Yaşargil reported one in 23 distal ACA aneurysms caused SDH $(4.3 \%))^{19)}$ No incidence of $\mathrm{SDH}$ associated aneurysm cases was quoted in other aneurysm series.

The prognosis for SDH caused by a ruptured aneurysm is grave. ${ }^{11,18)}$ Cases 1 and 3 suffered massive SDH and emergency surgery was of no avail in Case 1. Both patients were comatose and showed tentorial herniation signs on admission. Case 2 had significant SDH but it did not cause increased intracranial pressure. This patient recovered fully after aneurysmal clipping and returned to normal daily life. Weir et al. ${ }^{18)}$ reported only half of the 18 patients with aneurysm associated SDH survived and 13 patients showed tentorial herniation signs preoperative- 
ly. There is no doubt that good results are correlated with good admission grade in aneurysm cases with or without SDH.

\section{References}

1) Ban $\mathrm{S}$, Sato $\mathrm{S}$, Yamamoto $\mathrm{T}$, Ogata $\mathrm{M}$ : Distal anterior cerebral artery aneurysms causing acute subdural hematoma: Report of two cases. No Shinkei Geka 13: 911-916, 1985 (in Japanese)

2) Barton E, Tudor J: Subdural haematoma in association with intracranial aneurysm. Neuroradiology 23: $157-160,1982$

3) Bebin J, Currier RD: Cause of death in ruptured intracranial aneurysms. Arch Intern Med (Chicago) 99: 771-790, 1957

4) Boyd-Wilson JS: The association of cerebral angiomas with intracranial aneurysms. $J$ Neurol Neurosurg Psychial 22: 218-223, 1959

5) Clark E, Walton JN: Subdural haematoma complicating intracranial aneurysm and angioma. Brain 76: $378-404,1953$

6) Drake CG, Jory TA: Spontaneous intracranial haemorrhage: "Subarachnoid haemorrhage": A review of investigation and treatment in 189 cases. Canad J Surg 4: 4-11, 1960

7) Fein JM, Rovit RL: Interhemispheric subdural hematoma secondary to hemorrhage from a callosomarginal artery aneurysm. Neuroradiology 1: 183186,1970

8) Fox JL: Intracranial Aneurysms, vol 1. New York, Springer, 1983, pp 199-203

9) Golden J, Odom GL, Woodhall B: Subdural hematoma following subarachnoid hemorrhage. Arch Neurol Psychiat 69: 486-489, 1953

10) Handel SF, Perpetuo FOL, Handel CH: Subdural hematomas due to ruptured cerebral aneurysms: Angiographic diagnosis and potential pitfall for CT. AJR 130: 507-509, 1978

11) Hasegawa $T$, Komai $T$, Fujii $T$, Kashihara $K$, Ito $H$, Yamamoto $S$ : Computed tomography of acute subdural hematomas from intracranial aneurysmal rup- tures, as seen in relation to angiographic and clinical findings. CT Kenkyu 8: 645-652, 1986 (in Japanese)

12) Hazouri LA: A review of 75 intracranial aneurysms. $J$ Med Ass Georgia 44: 15-19, 1955

13) Honda E, Shojima K, Shigemori M, Kobayashi S, Higashi K, Hara K, Hayashi T: Two cases of ruptured cerebral aneurysm associated with acute subdural hematoma. Med Postgrad 22: 222-228, 1984 (in Japanese)

14) Kondziolka D, Berstein $M$, ter Brugge $K$, Schutz $H$ : Acute subdural hematoma from ruptured posterior communicating artery aneurysm. Neurosurgery 22: $151-154,1988$

15) O'Leary PM, Sweeny PJ: Ruptured intracerebral aneurysm resulting in a subdural hematoma. Ann Emerg Med 15: 944-946, 1986

16) Reichenthal E, Savitz MH, Rothman AS, Katz SS, Malis LI: Ruptured intracranial aneurysms as a cause of subdural haematoma. Potential diagnostic pitfalls and the surgical management of the acute patient. Neurochirurgia (Stuttg) 29: 219-224, 1986

17) Strauss I, Globus JH, Ginsburg SW: Spontaneous subarachnoid hemorrhage. Its relation to aneurysms of cerebral blood vessels. Arch Neurol Psychiat 27: 1080-1132, 1932

18) Weir B, Myles T, Kahn M, Maroun F, Malloy D, Benoit B, McDermott M, Cochrane D, Mohr G, Ferguson G, Durity F: Management of acute subdural hematomas from aneurysmal rupture. Can $J$ Neurol Sci 11: 371-376, 1984

19) Yaşargil MG: Microneurosurgery, vol I. Stuttgart, George Thieme, 1984, p 299

20) Yaşargil MG: Microneurosurgery, vol 11 . Stuttgart, George Thieme, 1984, p 225

Address reprint requests to: K. Watanabe, M.D., Department of Neurosurgery, Dokkyo University School of Medicine, 880 Kita-kobayashi, Mibu-machi, Shimotsuga-gun, Tochigi 321-02, Japan. 